\title{
Application of marine collagen for stem-cell-based therapy and tissue regeneration (Review)
}

\author{
CHAO LIU \\ Shanghai Ninth People's Hospital, Shanghai Jiao Tong University School of Medicine, Shanghai 200011, P.R. China
}

Received April 22, 2021; Accepted June 22, 2021

DOI: $10.3892 / \mathrm{mi} .2021 .5$

\begin{abstract}
Tissue engineering and regenerative medicine is becoming an important component in modern biological scientific research. Tissue engineering, a branch of regenerative medicine, is a field that is actively developing to meet the challenges presented in biomedical applications. This particularly applies to the research area of stem cells and biomaterials, due to both being pivotal determinants for the successful restoration or regeneration of damaged tissues and organs. Recently, the development of innovative marine collagen-based biomaterials has attracted attention due to the reported environmentally friendly properties, the lack of zoonotic disease transmission, biocompatibility, bioactivity, the lack of ethics-related concerns and cost-effectiveness for manufacturing. The present review aimed to summarize the potential application and function of marine collagen in stem cell research in a medical and clinical setting. In addition, the present review cited recent studies regarding the latest research advances into using marine collagen for cartilage, bone, periodontal and corneal regeneration. It also characterized the distinct advantages of using marine collagen for stem cell-based tissue repair and regeneration. In addition, the present review comprehensively discussed the most up to date information on stem cell biology, particularly the possibility of treating stem cells with marine collagen to maximize their multi-directional differentiation capability, which highlights the potential use of marine collagen in regenerative medicine. Furthermore, recent research progress on the potential immunomodulatory capacity of mesenchymal stem cells following treatment with marine collagen to improve the understanding of cell-matrix interactions was investigated. Finally, perspectives on the possible future research directions
\end{abstract}

Correspondence to: Dr Chao Liu, Shanghai Ninth People's Hospital, Shanghai Jiao Tong University School of Medicine, 639 Zhizaoju Road, Shanghai 200011, P.R. China

E-mail: liuchao968@126.com

Key words: fish collagen, tissue engineering, regenerative medicine, stem cells, biomaterial, cartilage regeneration, bone regeneration, periodontal regeneration, corneal regeneration, immunomodulatory for the application of marine collagen in the area of regenerative medicine are provided.

\section{Contents}

1. Introduction

2. Basic characteristics of marine collagen

3. Marine collagen and cartilage regeneration

4. Marine collagen and bone regeneration

5. Marine collagen and periodontal regeneration

6. Marine collagen and corneal regeneration

7. Marine collagen and neural regeneration

8. Marine collagen and oral mucosa regeneration

9. Marine collagen and their immunomodulatory properties in mesenchymal stem cells

10. Marine collagen and skin regeneration: Implications for wound dressing

11. Mechanism of marine collagen in tissue regeneration

12. Conclusions and future perspectives

\section{Introduction}

Tissue engineering and regenerative medicine is a rapidly evolving interdisciplinary field that integrates material science, bioengineering, medicine, cell biology, pharmacology and chemistry with the aim of regenerating compromised tissues and organs $(1,2)$. The most widely accepted concept of tissue engineering and regenerative medicine involves three main components: i) The development of functional and biocompatible scaffolds; ii) the selection of suitable stem cells; and iii) the induction of specific signaling pathways to repair or regenerate specific tissues or organs (3). Biomaterials are essential components for the development of scaffolds. The application of suitable biomaterials can improve stem cell adhesion, migration, proliferation and differentiation, which may promote their ability to restore and regenerate damaged tissues or organs (4). Stem cells are normally maintained in an undifferentiated state; however, they possess self-renewing, proliferative and multidirectional differentiation capacities, such that their offspring or daughter cells can develop into various lineages or cell types. These distinctive characteristics render stem cells an ideal candidate for use in regenerating injured tissues or organs $(2,5)$. Stem cells can be divided into 
different categories in a manner that is dependent on their origin and the differentiation signals they receive. Embryonic stem cells and induced pluripotent stem cells (iPSCs) are considered to be pluripotent, meaning that they can differentiate into any cell type in all three germ layers, namely the ectoderm, endoderm or mesoderm (5). By contrast, mesenchymal stem cells and hematopoietic stem cells are multipotent cells that can differentiate into any cell type only within a specific germ layer (5). In addition, mesenchymal stem cells possess immunoregulatory functions.

Among all naturally occurring polymers, collagen is one of the most extensively studied and applied in the clinical settings. Collagen accounts for $\sim 1 / 3$ of all proteins in an animal and is the major constituent of the extracellular matrix in connective tissues, including skin, bones, cartilage, ligaments and tendons (6). Collagen provides basic structural support to organs in all higher animals by contributing strength, elasticity and firmness, which are indispensable for effective tissue repair, regeneration and mobility mediated by intracellular mechanochemical signaling networks (7). Primary biomedical applications involving the use of collagen include biomaterial development, tissue engineering, absorbable surgical suture, hemostatic agents and burn/wound cover dressings (8). Due to the ethical or dietary implications in a social setting and the risk of animal disease transmission, such as bovine spongiform encephalopathy and foot-and-mouth disease, the scientific community is exploring the development of marine collagen as the substitute for mammalian collagen $(9,10)$. Another important reason for the preference of using marine collagen is that $\sim 75 \%$ of total fish weight is comprised of collagen (11), suggesting that there is an abundance of this type of collagen. On average, $\sim 3 / 4$ of a fish, including the skin, fins, the skeletal system and head are discarded during fish processing (12). The disposal of byproducts from fish processing can be economically burdensome and may pose a threat to environmental sustainability, since they are commonly dumped back into the ocean or disposed of at a landfill (13). Marine byproducts are important sources of valuable inorganic and organic materials which have potential for various industrial uses, such as in nutraceuticals, cosmetics and pharmaceuticals. The possible applications of marine collagen include, but are not limited to, tissue engineering, wound dressing, cosmetics and drug delivery $(12,14,15)$. A number of studies have previously found that hydrolyzed fish collagen exhibits biologically active characteristics, including anti-oxidative, immunomodulatory, anti-bacterial, anti-inflammatory and angiotensin-converting enzyme inhibitory properties (16-19). Therefore, the adequate application of fish collagen can not only limit the quantity of biowaste to protect the environment, but can also increase the economic value of fish byproducts.

The present review aimed to summarize the potential for the application of marine collagen in the field of tissue engineering and regenerative medicine. It focused on the recent research advances made in cartilage, bone, periodontal and corneal regeneration by using fish collagen. In particular, knowledge on the mechanisms underlying the regulation of stem cell physiology by marine collagen was summarized and highlighted. In addition, Tables I-III summarize the main content of the present review. Table I lists the application of marine collagen that has been previously reported/studied.
Table II lists studies that investigated stem cell proliferation and differentiation. Finally, Table III demonstrates the potential benefits and limitations of using marine collagen.

\section{Basic characteristics of marine collagen}

There are $\geq 28$ types of collagen in marine organisms (20). Type I collagen is the most abundant type of collagen and can be found in fish scales, skin and bone, whilst type II collagen can be found in the fish bone cartilage (21). Marine collagens exhibit a greater bioavailability and absorption efficiency in the body compared with mammal-derived collagen, such as bovine and porcine collagen (22). However, there are some disadvantages associated with the use of fish collagen, including higher rates of degradation, lower mechanical strength, low denaturation temperature and lower biomechanical stiffness (23). In addition, the thermal and structural stability of marine collagen are lower compared with those of mammalian collagen, which can be mainly ascribed to the lower proline and hydroxyproline content in marine collagen (24). Compared with mammalian collagen, marine collagens are more readily hydrolyzed by proteases (24). Since the mechanical property of marine collagen is the primary determinant of its biological activity, the lower mechanical strength and denaturation temperature of marine collagen results in the easier dissolution of collagen fibrils in physiological environments. This remains an obstacle for the biomedical application of marine collagen.

Pepsin or acid extraction procedures are usually used for collagen extraction. During pepsin extraction, the enzyme pepsin is used to cleave specific targets, such as the crosslink-containing telopeptides, which can enhance solubility and subsequent yield of collagen. The final product obtained using the pepsin extraction procedure is referred to as pepsin-soluble collagen. By contrast, hydrochloric or acetic acid can solubilize the collagen chains to improve the output capacity of the extraction procedure (8). The final product of acid extraction is referred to as acid-soluble collagen (8).

\section{Marine collagen and cartilage regeneration}

Cartilage defects are difficult to repair by themselves due to their avascular nature. The transplantation of mesenchymal stem cells is a favorable approach due to their high proliferative activity and their capacity to differentiate into chondrocytes, which are responsible for cartilage synthesis and maintenance (25). Numerous scaffolds that consist of synthetic and natural matrices have been applied either alone or with cells for the repair of cartilage defects. Natural, biodegradable scaffolds are mainly comprised of collagen, silk, agarose, chitosan, hyaluronic acid, alginate and gelatin. Natural polymers, such as fibrin and collagen, confer high levels of biocompatibility and can promote cell adhesion without incurring immune responses (26). In particular, marine collagen has been widely used for chondrogenic differentiation in the field of cartilage regeneration. The influence of hydrolyzed fish collagen on the chondrogenesis of equine adipose-derived stromal cells (ADSCs) has been previously investigated. In a previous study, hydrolyzed fish collagen was obtained from the skin of ocean fish (pollock, cod and haddock) (27). These types of fish collagen mainly consist of $\alpha 1$ and $\alpha 2$ chains arranged 
Table I. List of potential applications of marine collagen that have been reported/studied.

\begin{tabular}{llr}
\hline Collagen source & \multicolumn{1}{c}{ Field of research } & (Refs.) \\
\hline Fish skin (pollock cod and haddock) & Cartilage regeneration & $(27)$ \\
Jelly fish (Rhopilema esculentum and Rhizostoma pulmo) & Cartilage regeneration & $(28-30)$ \\
Chub skin (Hypophthalmichthys molitrix) & Cartilage regeneration \\
Blue Shark skin (Prionace glauca) & Cartilage regeneration \\
Tilapia scale & Cartilage regeneration \\
Tilapia scale & Bone regeneration \\
Tilapia skin & Bone regeneration \\
Atlantic salmon skin & Bone regeneration \\
Blue shark skin (Prionace glauca) & Bone regeneration \\
Mahi mahi fish bone(Coryphaena hippurus) & Bone regeneration \\
Salmon skin & Periodontal regeneration \\
Fish scale (Lates calcarifer) & Corneal regeneration \\
Tilapia fish scale $($ Oreochromis niloticas) & Corneal regeneration \\
Tilapia skin & Neural regeneration \\
\hline
\end{tabular}

Table II. List of studies that investigated stem cell proliferation or differentiation.

Equine adipose tissue-derived stromal cells

Human mesenchymal stem cells

Human mesenchymal stem cells

Mouse bone marrow-derived mesenchymal stem cells

Rat bone marrow-derived stem cells

Human adipose stem cells

Human mesenchymal stem cells

Human mesenchymal stem cells

Rat mesenchymal stem cells

Mouse bone marrow-derived mesenchymal stem cell

Human periodontal ligament fibroblasts
Chondrogenic differentiation

Chondrogenic differentiation

Osteogenic differentiation

Osteogenic differentiation

Proliferation

Proliferation and chondrogenic differentiation

Proliferation and chondrogenic differentiation

Proliferation and osteogenic differentiation

Proliferation, osteogenic and chondrogenic differentiation

Proliferation and osteogenic differentiation

Proliferation and osteogenic differentiation in a 2:1 ratio. The results of that study demonstrated that the extent of chondrogenic differentiation induced by hydrolyzed fish collagen was comparable to that induced by TGF- $\beta 1$, suggesting that hydrolyzed fish collagen alone can induce the chondrogenic differentiation of equine ADSCs (27).

Other studies instead have focused on collagen derived from jellyfish, which is an important component of the marine ecosystem. For example, collagen isolated from cured jellyfish Rhopilema esculentum by pepsin digestion has been reported to induce the chondrogenic differentiation of human mesenchymal stem cells (MSCs) (28). This was confirmed by the observed increased mRNA expression of collagen II, SRY-box transcription factor 9 (SOX9) and aggrecan along with a significantly increased sulfated glycosaminoglycan (GAG) deposition in the jellyfish collagen scaffolds after 21 days of cultivation (28). Another previous study investigated the potential of using hybrid scaffolds based on marine biomaterials, namely hydrogel alginate combined with jellyfish collagen from Rhopilema esculentum, for articular cartilage repair. This hybrid construct was found to uphold human mesenchymal stem cell chondrogenic differentiation and equip more stable constructs compared with pure hydrogels (29). Recently, a novel therapeutic implant for cartilage repair was developed by combining Rhizostoma Pulmo jellyfish type II collagen, TGF- $\beta 3$ and human bone marrow-derived mesenchymal stem cell (30). Reverse transcription-quantitative PCR and histological staining using Safranin-O/Fast Green, Alcian Blue and Alizarin Red staining suggested that the jellyfish type II collagen-containing implant induced chondrogenesis in human MSCs (30).

In addition to jellyfish, the skin of silver carp and blue shark in addition to tilapia scale, are other potential sources of collagen. Wang et al (31) prepared a nanocomposite film consisting of regenerated fish collagen extracted from the skin of Hypophthalmichthys molitrix, single-wall carbon nanotube-COOHs and TGF- $\beta 1$. The biocompatibility of these nanocomposite films was examined in vitro using Sprague Dawley rat bone marrow-derived stem cells, and the results 
Table III. Benefits and limitations of fish collagen application.

Benefits of fish collagen application

Limitations of fish collagen application

No ethical or dietary prohibitions

No transmission of land animal disease

Reduction in the quantity of marine waste

Reduction of environmental pollution

Increase in the economic value of fish byproducts

High bioavailability

High absorption efficiency
Higher degradation

Lower mechanical strength

Lower denaturation temperature indicated that the nanocomposite films could promote cell proliferation (31). In another recent study, the capability of blue shark (Prionace glauca) skin collagen to induce the chondrogenic differentiation of human adipose stem cells was investigated (32). The early chondrogenesis of human adipose stem cells treated with the shark collagen-based constructs was observed, which was corroborated by the markedly upregulated gene expression levels of chondrogenic markers, SOX9 and collagen II (32). The authors suggested that the Prionace glauca skin collagen itself may induce chondrogenesis at an early stage, although further exogenous stimulation may be required to sustain the phenotype (32). This suggests that Prionace glauca skin collagen may serve as an important component of highly effective matrices used for cartilage regeneration (32).

Apart from the aforementioned sources of marine collagen, tilapia scale can also be an excellent source of collagen. The chondrogenic differentiation ability of human MSCs cultured with tilapia scale collagen fibrils was previously examined (33). Human MSCs treated with tilapia scale collagen exhibited a higher GAG expression and stronger Safranin O staining (33). Furthermore, SOX9 expression at an earlier phase on day 4, and higher aggrecan and collagen II expression levels were observed in human MSCs cultured with tilapia collagen (33). Therefore, it is reasonable to hypothesize that tilapia scale may provide another applicable source of collagen for the chondrogenic differentiation of human MSCs (33).

\section{Marine collagen and bone regeneration}

Over the past decades, bone tissue engineering has become a promising strategy for the treatment of bone-related disorders, including osteoporosis and bone defects. The aim of bone tissue engineering is to promote bone regeneration through the coordinated integration of stem cells, biomaterials and bone-inducing factors. Effective stem cell-based bone tissue engineering requires a combination of inducers, sufficient mesenchymal progenitors with osteogenic properties and biocompatible materials to drive osteogenic differentiation (34).

Biomaterials serve as the basic component of scaffolds in bone tissue engineering. Typical bone biomaterials that have been used as scaffolds for bone tissue engineering include natural/synthetic polymers, bioactive ceramics, decellularized extracellular matrices, biodegradable metals or composites of any those aforementioned (35). Among these, fish collagen has been attracting substantial research interest as an adequate alternative to mammalian collagen for bone tissue engineering (36-38). Tilapia is an important farmed freshwater species of fish that has become one of the most popular sources of collagen for bone tissue engineering. The impact of tilapia fish scale collagen fibril formation on the osteoblastic differentiation ability of human MSCs has previously been investigated (39). In that previous study, human MSCs were found to easily adhere to tilapia scale collagen, which significantly advanced early-stage osteoblastic differentiation in human MSCs (39). The levels of osteogenic-related markers, such as alkaline phosphatase (ALP) activity, bone morphogenetic protein 2 and osteopontin, were all revealed to be significantly upregulated when the human MSCs were cultured on the tilapia fish collagen surface, particularly during the initial phase of osteogenesis (39). Recently, three-dimensional tilapia scale collagen scaffolds have been produced using a microbial transglutaminase (mTGase) cross-linking method (40). Human MSCs in this scaffold had increased osteopontin expression levels and higher alkaline phosphatase activity compared with those in human MSCs in other scaffolds (40). The tilapia scale collagen scaffold crosslinked by mTGase exerted a more prominent effect on the osteogenesis process, suggesting that this combination of tilapia scale collagen and mTGase may be potentially used for bone tissue engineering (40). In addition, type I collagen has been previously extracted from tilapia fish skin and incorporated into natural bone-like three-dimensional-matrices. The effect of this matrix on bone homeostasis was assessed using mouse bone marrow-derived MSCs. After 14 days, a higher cell differentiation rate was observed in the mouse bone marrow-derived MSCs cultured on the fish collagen-based matrix, where their cellular calcium levels progressively increased with increasing culture duration (41).

Salmon is a highly valued species of fish that has been more extensively studied compared with other fish species (42). It is also one of the most important sources of collagen. In a previous study, soluble type I-like collagen collected from Atlantic salmon skin was used to construct porous scaffolds before cytocompatibility evaluation was performed using human MSCs. Human MSCs were able to adhere to these scaffolds, where the cell number raised during treatment (43). Furthermore, the osteogenic differentiation of these MSCs was confirmed based on the results of ALP activity assay (43). 
Raftery et al (44) previously investigated whether salmon skin collagen can serve as a substitute for bovine tendon collagen for tissue engineering applications. Primary rat MSCs were used in that study, where this type salmon skin collagen was found to be comparable to mammalian collagen (44).

The blue shark (Prionace glauca) exhibits the widest distribution range and is the most abundant species of shark that is hunted by fisheries (45). Collagen from the blue shark has also been assessed for the fabrication of biomaterials owing to its bioactivity and mechanical properties (46). Recently, collagen was extracted from blue shark skin before its biochemical and osteogenic properties were examined. The proliferation rate of the differentiated mouse bone marrow-derived MSCs was increased in a dose-dependent manner in the collagen-treated groups. In addition, higher mRNA and protein expression levels of osteogenic markers were observed in the collagen-treated groups, suggesting that the collagen extracted from blue shark skin possesses good osteogenic and biochemical properties and may serve as a good candidate for biological applications (47).

In addition to the collagen that can be extracted from tilapia, salmon and blue sharks, other fish collagen has also been used in the biomaterial field for bone tissue engineering. For example, hydrolyzed collagen has been prepared directly from Mahi mahi fish (Coryphaena hippurus) bones before its osteogenic capacity was investigated using mouse bone marrow-derived MSCs (48). The results of that previous study revealed that the expression of osteogenesis markers in mouse bone marrow-derived MSCs, namely ALP, collagen and osteocalcin, were significantly increased upon hydrolyzed Mahi mahi fish collagen treatment (48). This indicated that hydrolyzed fish collagen possesses excellent osteogenic effects and may be a useful biomaterial for bone regeneration (48).

\section{Marine collagen and periodontal regeneration}

Periodontal disease is a chronic inflammatory disease that can lead to the disconnection of periodontal ligament-like tissues, irreversible periodontal complex destruction, and the subsequent loss of barrier function and teeth (49). In recent years, regenerative strategies that can facilitate periodontal tissue repair have been investigated. This approach was focused on the combination of biomaterials, growth factors and stem cells to attain periodontal regeneration (50). As such, biomaterials can provide templates to facilitate the regeneration of periodontal tissues. Numerous types of biomaterials, such as synthetic organic materials (examples include poly-lactic-co-glycolic acid and poly-lactic acid) and natural organic materials (examples include collagen, silk fibroin, chitosan and alginate), have been extensively investigated for the possibility of periodontal regeneration (51).

Due to the reduced risk of zoonotic disease transmission and the readily available reservoir of industrial fish waste from which collagen can be extracted, fish collagen has attracted attention over the years for use in the production of tissue-engineered biomaterials for periodontal regeneration (52-54). A previous study produced a crosslinked collagen gel with acid-soluble collagen that was obtained from salmon skin. The growth rate and differentiation capacity of human periodontal ligament fibroblasts (HPdLFs) cultured on this salmon collagen gel were then examined (55). The growth rate was found to be higher when these cells were cultured on the salmon collagen gel compared with that in cells cultured on the porcine collagen gel (55). In addition, the HPdLFs cultured on the salmon collagen gel exhibited a higher ALP activity than those cultured on the porcine collagen gel, and a higher mRNA expression of ALP, collagen type I and osteocalcin was also observed (55). In another study that assessed the performance of a scaffold based on the salmon collagen-coated ePTFE, it was found that the outgrown cells penetrated the coated mesh fiber networks and formed complex multicellular layers (56). Moreover, an elevated ALP activity in response to osteogenesis induction was observed. In vitro mineralization was markedly increased in the primary tissue segment regions, along with some micro-mineral deposits formed on the coated-fiber networks. These findings suggest that the complex three-dimensional mesh consisting of salmon collagen-coated fibers considerably enhanced the osteogenesis of human periosteal sheets and biological mineralization (56).

\section{Marine collagen and corneal regeneration}

In total, 10 million individuals worldwide are affected by corneal-related blindness (57). Full or partial corneal transplantation is typically the only effective treatment to restore vision. The limited availability of corneal tissues due to donor shortages has inspired researchers to search for an alternative treatment to transplantation. It has been previously reported that the following three aspects can influence the regeneration of damaged tissues in limbal tissue engineering: Bioactive scaffolds, cell availability and suitable growth factors, similar to other tissues (58). Among the bioactive scaffolds, collagen matrices, amniotic membranes and hydrogels, along with natural polymers, have been previously studied (58). Since the human corneal stroma is rich in type I collagen, collagen has been postulated to be an ideal material for corneal scaffolds (59). One important source of collagen is fish scales, whereby scales of tilapia cultured under controlled conditions mainly consist of type I collagen that align in a manner identical to that observed in human corneas (59). As a result, this type of fish scale collagen may offer a viable and economical substitute that can facilitate corneal regeneration (59). In a previous study, a scaffold with collagen was collected from the fish scales of Lates calcarifer for corneal tissue engineering before its physiological, mechanical and cultural characteristics in a scaffold were evaluated following co-culture with corneal cells (60). The cultured corneal cells were examined by reverse transcription-quantitative PCR for putative stem cell markers. The results of that study confirmed that the cultured cells were of the limbal phenotype. Moreover, corneal cell differentiation was confirmed by the expression of marker genes, including keratin 3 and keratin 12 (60). In another previous study, to investigate whether fish scale-derived collagen matrices (FSCM) can meet the required standard to be a component of the reconstructed cornea, collagen matrices were prepared from tilapia fish scales (Oreochromis niloticas) (59). This fish scale-derived collagen matrix was used to grow corneal 
epithelial and stromal cells. No cytotoxicity was observed and primary human corneal epithelial cells cultured on the fish scale-derived collagen matrix stained positive for cytokeratin 3/12 and the stem cell marker p63, suggesting that the correct phenotype of primary human corneal epithelial cells was obtained following culture on this FSCM (59).

\section{Marine collagen and neural regeneration}

Neural tissue engineering is primarily focused on applying novel methods to recover functions in the nervous system. A biomaterial is a key component of the final complex used for neural tissue engineering, which has become crucial not only for the research of disease pathogenesis, but also for its potential application for tissue engineering (61). Various biomaterials, including collagen, have been applied in neural tissue engineering (62). Recently, marine collagen has attracted attention in the field of neural tissue engineering due to its excellent reported bioactivity and biocompatibility. Iwashita et al (63) isolated tilapia skin collagen gels to investigate its applicability for stem cell culture, whilst also assessing the influence of tilapia skin collagen gel stiffness on neural differentiation. iPSCs were used in these experiments, where the results revealed that the neural characteristics acquired after 5 days of neural induction on a tilapia collagen gel at a stiffness of $1,500 \mathrm{~Pa}$ switched to the lineage of dorsal forebrain neurons (63). These findings imply that tilapia collagen gel can be used for the neural induction of pluripotent stem cells (PSCs) (63).

\section{Marine collagen and oral mucosa regeneration}

In addition to cartilage, bone, periodontal, corneal and neural regeneration, marine collagen has also been considered for use in other areas of regenerative medicine. For example, recently, a fish scale collagen-derived scaffold with various microstructure prototypes was constructed that mimicked the dermal-epidermal junction of the oral mucosa. Expression of stem cell markers in oral keratinocytes was examined by immunohistochemistry. A fully differentiated, stratified epithelial layer was demonstrated to be developed within the scaffold, indicating that the micro-structured fish scale collagen scaffold can be used to produce tissue-engineered oral mucosal equivalents for clinical application (64).

\section{Marine collagen and their immunomodulatory properties in mesenchymal stem cells}

The immune response is essential for fighting infections and eliminating pathogens during the early stages of tissue regeneration and wound healing. In particular, Toll-like receptors belong to an important family of pattern recognition receptors that are involved in the immune response. They recognize signals from damaged extracellular matrix or necrotic tissues to activate tissue-resident macrophages and recruit other immune cells, including lymphocytes (such as B and T lymphocytes) and neutrophils. Neutrophils generate antibacterial substances and secrete inflammatory cytokines that support angiogenesis to facilitate the recovery of tissues. However, persistent and/or excessive inflammatory responses can lead to the development of chronic wounds and failure of tissue regeneration (65). In addition, the immune response during tissue regeneration is dependent on the subclass of macrophages activated. $\mathbf{M}_{2}$-type macrophages are helpful for wound healing, whilst the pro-inflammatory $\mathrm{M}_{1}$-type of macrophages exasperate tissue damage (66). The induction of macrophage subtype polarization from $\mathrm{M}_{1}$ to $\mathrm{M}_{2}$ has been found to be an effective treatment method in tissue engineering and regenerative medicine by alleviating the excessive inflammatory response $(67,68)$. By contrast, the immunoregulatory function of MSCs has emerged as a unique research hotspot and direction of clinical application. Along with their immunophenotype differentiation potential, multipotent mesenchymal stromal/stem cells display distinct immunomodulatory paracrine properties. Mesenchymal stromal/stem cells can inhibit the function of various types of mature immune cells through both secretory and cell-cell contact mechanisms $(69,70)$. For example, mesenchymal stem/stromal cells can modify immunological reactions through $\mathrm{T}$ cell suppression and by causing a shift in macrophages from the $M_{1}$ to $M_{2}$ phenotype (71).

The immune system can be divided into two parts: The innate immune system and the adaptive immune system. MSCs can interact extensively with cells that belong to both arms of the immune system. Specifically, in the innate immune system, MSCs can interact with macrophages, dendritic cells and natural killer (NK) cells. MSCs can not only exert immunosuppressive effects by inhibiting the generation of inflammatory cytokines, but can also promote the production of anti-inflammatory factors. In macrophages, MSCs can prevent polarization into the pro-inflammatory $\mathrm{M}_{1}$ phenotype, which can produce inflammatory mediators, including TNF- $\alpha$, IL-6, IL-1 $\beta$ and nitric oxide $(72,73)$. Additionally, MSCs can promote macrophage polarization into the anti-inflammatory $\mathrm{M}_{2}$ phenotype, and these macrophages which produce anti-inflammatory factors, such as TGF- $\beta$ and IL-10 (72,73). MSCs can also inhibit the function and maturation of dendritic cells by inhibiting the expression of maturation markers, including major histocompatibility complex (MHC)-II CD86 (74). In NK cells, MSCs can suppress IL-2-induced proliferation and inhibit the cytotoxic activity of cytokines (75).

In the adaptive or specialized immune system, there is a dynamic association between MSCs and cells in the adaptive immune system, such as T and B cells. MSCs can inhibit $\mathrm{T}$ cell proliferation and suppress the secretion of IFN $-\gamma$ by Th1 cells, whilst enhancing the secretion of IL- 4 by Th2 cells, which induces transformation from the Th1 to the Th2 subtype $(76,77)$. Using a co-culture system of MSCs and B cells, it was previously found that the differentiation of B cells into plasma cells was suppressed by MSCs, which led to the decreased production level of IgG1 and IgM (78).

Both stem cells and biomaterials can modulate the immune microenvironment during tissue regeneration. In addition, biomaterials can also exert effects on the immunoregulatory property of MSCs. It has been found that MSCs can sustain the expression of the key immunomodulatory molecules, B7-H3/CD276 and MHC, class I, E when subjected to osteogenic, adipogenic and chondrogenic differentiation in vitro (79). Therefore, understanding the 
effects of biomaterials on the immunoregulatory properties of MSCs is becoming an increasingly important question to address $(80,81)$. Recently, Liu and Sun (82) established a direct contact co-culture model consisting of hydrolyzed fish collagen treated-bone marrow-derived MSCs and RAW264.7 macrophages. Hydrolyzed fish collagen-induced bone marrow-derived MSCs were found to inhibit the expression and secretion of IL- 6 and IL-1 $\beta$, whilst increasing the levels of inflammatory zone 1 (FIZZ1) and CD206 in macrophages. Furthermore, hydrolyzed fish collagen-treated BMSCs also increased the secretion of TGF- $\beta$ and IL-10 by macrophages. These observations were shown to be mediated by the prostaglandin E2/prostaglandin receptor EP4-C/CCAAT-enhancer-binding protein axis. This suggested that osteogenically differentiated MSCs treated with hydrolyzed fish collagen retained their immunomodulatory properties (82). However, only a limited number of studies, as those aforementioned, have examined the effects of biomaterials on the immunoregulatory properties of MSCs. Considering the probable immunomodulatory role of MSCs, further investigations are required to shed more light on the effects of marine collagen on the immunomodulatory property of MSCs.

\section{Marine collagen and skin regeneration: Implications for wound dressing}

Skin plays a key role as the primary barrier for the body and protects it from the environment. During skin defects or when a wound is created, appropriate wound dressing is required to provisionally cover the wound area and accelerate wound healing (83). The ideal wound dressing should retain moisture, absorb wound exudates and protect the damaged area from secondary infections.

The conventional paraffin gauze dressing is generally used for clinic settings. However, some inherent disadvantages exist, such as low water absorption. This frequently leads to excess exudate leakage, which hinders the wound healing process and increases the risk of infection. In recent years, fish collagen had been increasingly used for wound dressing applications due to their low antigenicity, biodegradability and biocompatibility (84-86). Recently, collagen extracted from Hypophthalmichthys nobilis skin has been used to serve as the main component for wound dressing, which has been found to have good swelling properties in in vitro settings and exhibits antioxidant activity (87). In addition, this type of wound dressing does not cause erythrocyte hemolysis and sufficiently supports cell proliferation (87).

In another previous study, collagen sponges were prepared from type I collagen isolated from the skin of freshwater fish. By combining with rifampin, a fish collagen-based wound dressing was produced. This type of wound dressing demonstrated good mechanical properties and exhibited desirable biocompatibility, since the cells penetrated into the sponge and virtually no signs of local irritation were observed (88). Recently, collagen from the fish scale of Larimichthys crocea was extracted and a hybrid hydrogel based on this collagen was developed for wound healing (89). This hybrid hydrogel was found to be effective against bacterial infection by species, including Staphylococcus aureus and
Escherichia coli, in addition to enhancing cell proliferation and angiogenesis in vitro. Furthermore, fish collagen-based dressing could enhance full-thickness wound healing in a rat model by promoting angiogenesis, collagen deposition and re-epithelialization (89). Similarly, tilapia fish skin collagen (Oreochromis niloticus) was extracted to prepare a collagen hydrogel for the treatment of refractory wounds (90). Skin repair experiments and pathological analysis demonstrated that this type of wound dressing based on fish collagen could significantly promote the healing of deep second-degree burn wounds and the regeneration of skin appendages (90).

\section{Mechanism of marine collagen in tissue regeneration}

The quality of collagen depends on its rheological, functional and biochemical properties, which is dependent on the manufacturing method applied and the original fish species (91). Furthermore, the bioactive potential of marine collagen is profoundly influenced by its arrangement and molecular composition, which is varied among the different fish species. For example, the quantity of hydroxyproline content and amino acid composition in the marine collagen may depend on the body temperature and seasonal variations of the fish species. By contrast, the molecular composition of marine collagen, including the amino acid composition and arrangement, is different among collagens obtained by different extraction methods. It has been reported that hydrolyzed fish collagen is an effective differentiation stimulant for the chondrogenic lineage, where its regulatory effects have been proposed to be mediated by its triple-helical structure (27). The triple helix in collagen is established to possess a GFOGER peptide sequence on its surface, which can interact with $\alpha 10 \beta 1, \alpha 11 \beta 1, \alpha 1 \beta 1, \alpha 2 \beta 1$ and $\alpha 1$ integrins on the surfaces of cells to serve as a pseudo-ligand for mechanical signaling transduction (92). The effect of marine collagen is also dependent on its composition. For example, Sewing et al (93) proposed that due to the homo-trimeric composition of collagen from jellyfish, this collagen closely resembled that of vertebrate collagen type II, rendering this type of jelly fish collagen suitable for the cultivation of chondrocytes. It has also been suggested that certain amino acids, including glycine, alanine, glutamine and asparagine of collagen can specifically trigger osteogenesis through the activation of the focal adhesion kinase/JNK signaling pathway and runt-related transcription factors in bone marrow-derived MSCs (94). The low content of hydroxyl groups in salmon collagen leads to higher hydrophobicity, which repels water. Therefore, it is reasonable to suggest that the hydrophobicity of salmon collagen would affect its ability to attach to cells and would also affect cell morphology. In addition, the amino acid sequence of the collagen peptide can also influence cell attachment and adhesion (55). The interconnective micro-porous structures of marine based collagen can affect the direction of stem cell differentiation (32). Furthermore, the mechanical performance of marine collagen has been recognized to regulate cell morphology and intracellular functions. Nagai et al (55) indicated that the high mechanical strength of salmon collagen may impact on the proliferation and ALP activities of HPdLFs (55). In addition, early osteogenic 
induction may be facilitated by the increased rates of fibril formation by tilapia scale collagen (39).

\section{Conclusions and future perspectives}

In the present review, the possible application of marine collagen in the area of tissue engineering and regenerative medicine was focused upon, with particular emphasis on the induction of stem cells for cartilage, bone, periodontal and corneal regeneration. The information presented herein may facilitate the deeper understanding of the interaction between marine collagen and stem cells. The future application of marine collagen in tissue engineering and regenerative medicine is promising due to its inherent advantages, such as biocompatibility and environmental friendliness $(95,96)$. However, there are also certain disadvantages, including rapid degradation and low mechanical strength (87). Therefore, the further understanding of the modification methods used for improving the physicochemical properties of marine collagen and the development of novel manufacturing technologies are required. In addition, further knowledge is needed concerning the immunoregulatory properties of marine collagen. The potential scope of application for fish collagen in stem cell therapy remains narrow at present. It is mainly used for modulating MSCs to date, although the potential application of marine collagen for manipulating iPSC and embryonic stem cell physiology requires further investigation. Finally, the mechanisms underlying the effect of marine collagen on the stem cells warrant further examination, particularly in in vivo settings. Despite all the aforementioned challenges, marine collagen holds great promise in the field of novel biomaterial development for biomedicine.

\section{Acknowledgements}

Not applicable.

\section{Funding}

The present study was supported by the National Natural Science Foundation of China (grant no. 31600760).

\section{Availability of data and materials}

Not applicable.

\section{Author's contributions}

CL was involved in the conception and design of the study. CL also obtained and supervised funds, and wrote, drafted, revised, edited and reviewed the manuscript. The author CL confirms the authenticity of all the raw data. The author has read and approved the final manuscript.

\section{Ethics approval and consent to participate}

Not applicable.

\section{Patient consent for publication}

Not applicable.

\section{Competing interests}

The author declares that he has no competing interests.

\section{References}

1. Caddeo S, Boffito M and Sartori S: Tissue engineering approaches in the design of healthy and pathological in vitro tissue models. Front Bioeng Biotechnol 5: 40, 2017.

2. Cossu G, Birchall M, Brown T, De Coppi P, Culme-Seymour E, Gibbon S, Hitchcock J, Mason C, Montgomery J, Morris S, et al: Lancet commission: Stem cells and regenerative medicine. Lancet 391: 883-910, 2018.

3. La Noce M,Paino F, Spina A, Naddeo P, Montella R, Desiderio V, De Rosa A, Papaccio G, Tirino V and Laino L: Dental pulp stem cells: State of the art and suggestions for a true translation of research into therapy. J Dent 42: 761-768, 2014.

4. Tsutsui TW: Dental pulp stem cells: Advances to applications. Stem Cells Cloning 13: 33-42, 2020.

5. Glotzbach JP, Wong VW, Gurtner GC and Longaker MT: Regenerative medicine. Curr Probl Surg 48: 148-212, 2011.

6. Subhan F, Hussain Z, Tauseef I, Shehzad A and Wahid F: A review on recent advances and applications of fish collagen. Crit Rev Food Sci Nutr 61: 1027-1037, 2021.

7. Tziveleka LA, Ioannou E, Tsiourvas D, Berillis P, Foufa E and Roussis V: Collagen from the marine sponges Axinella Cannabina and Suberites Carnosus: Isolation and morphological, biochemical, and biophysical characterization. Mar Drugs 15: $152,2017$.

8. Jafari H, Lista A, Siekapen MM, Ghaffari-Bohlouli P, Nie L, Alimoradi $\mathrm{H}$ and Shavandi A: Fish collagen: Extraction, characterization, and applications for biomaterials engineering. Polymers (Basel) 12: 2230, 2020.

9. Adamiak K, Lewandowska K and Sionkowska A: The infuence of salicin on rheological and film-forming properties of collagen. Molecules 26: 1661, 2021.

10. Liu C, Liu X, Xue Y, Ding TT and Sun J: Hydrolyzed tilapia fish collagen modulates the biological behavior of macrophages under inflammatory conditions. RSC Adv 5: 30727-30736, 2015.

11. Senaratne LS, Park PJ and Kim SK: Isolation and characterization of collagen from brown backed toadfish (Lagocephalus gloveri) skin. Bioresour Technol 97: 191-197, 2006.

12. Alves A, Marques A, Martins E, Silva T and Reis R: Cosmetic potential of marine fish skin collagen. Cosmetics 4: 39, 2017.

13. Muralidharan N, Jeya Shakila R, Sukumar D and Jeyasekaran G: Skin, bone and muscle collagen extraction from the trash fish, leather jacket (Odonus niger) and their characterization. J Food Sci Technol 50: 1106-1113, 2013.

14. Aguirre-Cruz G, León-López A, Cruz-Gómez V, Jiménez-Alvarado R and Aguirre-Álvarez G: Collagen hydrolysates for skin protection: Oral administration and topical formulation. Antioxidants (Basel) 9: 181, 2020.

15. Liu C, Xue Y and Sun J: Hydrolyzed fish collagen inhibits inflammatory cytokines secretion in lipopolysaccharide-induced HUVECs. Adv Materials Res 1025-1026: 570-573, 2014.

16. Nurilmala M, Hizbullah HH, Karnia E, Kusumaningtyas E and Ochiai Y: Characterization and antioxidant activity of collagen, gelatin, and the derived peptides from yellowfin tuna (Thunnus albacares) Skin. Mar Drugs 18: 98, 2020.

17. Liu C and Sun J: Marine based biomaterial-fish collagen enhances the polarization of human macrophage. IOP Conf Ser Mater Sci Eng 1040: 012006, 2021.

18. Guo L, Harnedy PA, Zhang L, Li B, Zhang Z, Hou H, Zhao X and FitzGerald RJ: In vitro assessment of the multifunctional bioactive potential of Alaska pollock skin collagen following simulated gastrointestinal digestion. J Sci Food Agric 95: 1514-1520, 2015.

19. Liu $C$ and Sun J: Fish collagen promotes proliferation and inhibits the inflammation in human epidermal keratinocytes in vitro. BIBE2020: In: Proceedings of the Fourth International Conference on Biological Information and Biomedical Engineering. Association for Computing Machinery, New York, NY, 2020.

20. Meyer M: Processing of collagen based biomaterials and the resulting materials properties. Biomed Eng Online 18: 24, 2019.

21. Cumming MH, Hall B and Hofman K: Isolation and characterisation of major and minor collagens from hyaline cartilage of hoki (Macruronus novaezelandiae). Mar Drugs 17: 223, 2019. 
22. Sripriya R and Kumar R: A novel enzymatic method for preparation and characterization of collagen film from swim bladder of fish rohu (Labeo rohita) Food Nutr Sci 6: 1468-1478, 2015.

23. Subhan F, Ikram M, Shehzad A and Ghafoor A: Marine Collagen: An emerging player in biomedical applications. J Food Sci Technol 52: 4703-4707, 2015.

24. Avila Rodríguez MI, Rodríguez Barroso LG and Sánchez ML: Collagen: A review on its sources and potential cosmetic applications. J Cosmet Dermatol 17: 20-26, 2018

25. Bornes TD, Adesida AB and Jomha NM: Mesenchymal stem cells in the treatment of traumatic articular cartilage defects: A comprehensive review. Arthritis Res Ther 16: 432, 2014.

26. Armiento AR, Stoddart MJ, Alini M and Eglin D: Biomaterials for articular cartilage tissue engineering: Learning from biology. Acta Biomater 65: 1-20, 2018.

27. Raabe O, Reich C, Wenisch S, Hild A, Burg-Roderfeld M Siebert HC and Arnhold S: Hydrolyzed fish collagen induced chondrogenic differentiation of equine adipose tissue-derived stromal cells. Histochem Cell Biol 134: 545-554, 2010.

28. Hoyer B, Bernhardt A, Lode A, Heinemann S, Sewing J, Klinger M, Notbohm $\mathrm{H}$ and Gelinsky M: Jellyfish collagen scaffolds for cartilage tissue engineering. Acta Biomater 10: 883-892, 2014

29. Pustlauk W, Paul B, Gelinsky M and Bernhardt A: Jellyfish collagen and alginate: Combined marine materials for superior chondrogenesis of hMSC. Mater Sci Eng C Mater Biol Appl 64: 190-198, 2016.

30. Pugliano M, Vanbellinghen X, Schwinté P, Benkirane-Jessel N and Keller L: Combined jellyfish collagen type ii, human stem cells and tgf-B3 as a therapeutic implant for cartilage repair. J Stem Cell Res Ther 7: 2, 2017.

31. Wang J, He C, Cheng N, Yang Q, Chen M, You L and Zhang Q: Bone marrow stem cells response to Collagen/Single-Wall carbon nanotubes-COOHs nanocomposite films with transforming growth factor beta 1. J Nanosci Nanotechnol 15: 4844-4850, 2015.

32. Diogo GS, Carneiro F, Freitas-Ribeiro S, Sotelo CG, Pérez-Martín RI, Pirraco RP, Reis RL and Silva TH: Prionace glauca skin collagen bioengineered constructs as a promising approach to trigger cartilage regeneration. Mater $\mathrm{Sci}$ Eng C Mater Biol Appl 120: 111587, 2021.

33. Hsu HH, Uemura T, Yamaguchi I, Ikoma T and Tanaka J: Chondrogenic differentiation of human mesenchymal stem cells on fish scale collagen. J Biosci Bioeng 122: 219-225, 2016.

34. Pereira HF, Cengiz IF, Silva FS, Reis RL and Oliveira JM: Scaffolds and coatings for bone regeneration. J Mater Sci Mater Med 31: 27, 2020.

35. Safari B, Aghanejad A, Roshangar L and Davaran S: Osteogenic effects of the bioactive small molecules and minerals in the scaffold-based bone tissue engineering. Colloids Surf B Biointerfaces 198: 111462, 2021.

36. Jin S, Sun F, Zou Q, Huang J, Zuo Y, Li Y, Wang S, Cheng L, Man Y, Yang F and Li J: Fish collagen and hydroxyapatite reinforced poly(lactide-co-glycolide) Fibrous membrane for guided bone regeneration. Biomacromolecules 20: 2058-2067, 2019.

37. Liu C and Sun J: Potential application of hydrolyzed fish collagen for inducing the multidirectional differentiation of rat bone marrow mesenchymal stem cells. Biomacromolecules 15 : 436-443, 2014

38. Oh GW, Nguyen VT, Heo SY, Ko SC, Kim CS, Park WS, Choi IW and Jung WK: 3D PCL/fish collagen composite scaffolds incorporating osteogenic abalone protein hydrolysates for bone regeneration application: In vitro and in vivo studies. J Biomater Sci Polym Ed 32: 355-371, 2021.

39. Matsumoto R, Uemura T, Xu Z, Yamaguchi I, Ikoma T and Tanaka J: Rapid oriented fibril formation of fish scale collagen facilitates early osteoblastic differentiation of human mesenchymal stem cells. J Biomed Mater Res A 103: 2531-2539, 2015 .

40. Oh HH, Uemura T, Yamaguchi I, Ikoma T and Tanaka J: Effect of enzymatically cross-linked tilapia scale collagen for osteoblastic differentiation of human mesenchymal stem cells. J Bioactive Compatible Polymers 31: 31-41, 2016.

41. Elango J, Saravanakumar K, Rahman SU, Henrotin Y, Regenstein JM, Wu W and Bao B: Chitosan-Collagen 3D matrix mimics trabecular bone and regulates RANKL-Mediated paracrine cues of differentiated osteoblast and mesenchymal stem cells for bone marrow macrophage-Derived osteoclastogenesis. Biomolecules 9: 173, 2019.
42. Hodgson EE, Wilson SM and Moore JW: Changing estuaries and impacts on juvenile salmon: A systematic review. Glob Chang Biol 26: 1986-2001, 2020.

43. Hoyer B, Bernhardt A, Heinemann S, Stachel I, Meyer M and Gelinsky $\mathrm{M}$ : Biomimetically mineralized salmon collagen scaffolds for application in bone tissue engineering. Biomacromolecules 13: 1059-1066, 2012.

44. Raftery RM, Woods B, Marques ALP, Moreira-Silva J, Silva TH, Cryan SA, Reis RL and O'Brien FJ: Multifunctional biomaterials from the sea: Assessing the effects of chitosan incorporation into collagen scaffolds on mechanical and biological functionality. Acta Biomater 43: 160-169, 2016.

45. Bailleul D, Mackenzie A, Sacchi O, Poisson F, Bierne N and Arnaud-Haond S: Large-scale genetic panmixia in the blue shark (Prionace glauca): A single worldwide population, or a genetic lag-time effect of the 'grey zone' of differentiation? Evol Appl 11: 614-630, 2018.

46. Blanco M, Sanz N, Valcarcel J, Pérez-Martín RI and Sotelo CG: Does Subunit Composition influence the intermolecular crosslinking of fish collagen? A Study with hake and blue shark skin collagens. Polymers (Basel) 12: 1734, 2020.

47. Elango J, Lee JW, Wang S, Henrotin Y, de Val JEMS, M Regenstein J, Lim SY, Bao B and Wu W: Evaluation of differentiated bone cells proliferation by blue shark skin collagen via biochemical for bone tissue engineering. Mar Drugs 16: 350, 2018.

48. Elango J, Robinson J, Zhang J, Bao B, Ma N, de Val JEMS and Wu W: Collagen peptide Upregulates Osteoblastogenesis from bone marrow mesenchymal stem cells through MAPK-Runx2. Cells 8: 446, 2019.

49. d'Avanzo N, Bruno MC, Giudice A, Mancuso A, Gaetano F, Cristiano MC, Paolino D and Fresta M: Influence of materials properties on bio-physical features and effectiveness of 3D-Scaffolds for periodontal regeneration. Molecules 26: 1643 , 2021.

50. Park CH: Biomaterial-based approaches for regeneration of periodontal ligament and cementum using $3 \mathrm{D}$ platforms. Int J Mol Sci 20: 4364, 2019

51. Matichescu A, Ardelean LC, Rusu LC, Craciun D, Bratu EA, Babucea $\mathrm{M}$ and Leretter $\mathrm{M}$ : advanced biomaterials and techniques for oral tissue engineering and regeneration-a review. Materials (Basel) 13: 5303, 2020.

52. Yunoki S, Nagai N, Suzuki T and Munekata M: Novel biomaterial from reinforced salmon collagen gel prepared by fibril formation and cross-linking. J Biosci Bioeng 98: 40-47, 2004.

53. Liu C and Sun J: Hydrolyzed tilapia fish collagen induces osteogenic differentiation of human periodontal ligament cells. Biomed Mater 10: 065020, 2015.

54. Zhou T, Liu X, Sui B, Liu C, Mo X and Sun J: Development of fish collagen/bioactive glass/chitosan composite nanofibers as a GTR/GBR membrane for inducing periodontal tissue regeneration. Biomed Mater 12: 055004, 2017.

55. Nagai N, Mori K, Satoh Y, Takahashi N, Yunoki S, Tajima K and Munekata M: In vitro growth and differentiated activities of human periodontal ligament fibroblasts cultured on salmon collagen gel. J Biomed Mater Res A 82: 395-402, 2007.

56. Kawase T, Okuda K, Kogami H, Nakayama H, Nagata $M$ and Yoshie H: Osteogenic activity of human periosteal sheets cultured on salmon collagen-coated ePTFE meshes. J Mater Sci Mater Med 21: 731-739, 2010

57. Tidu A, Schanne-Klein MC and Borderie VM: Development, structure, and bioengineering of the human corneal stroma: A review of collagen-based implants. Exp Eye Res 200: 108256, 2020.

58. Mobaraki M, Abbasi R, Omidian Vandchali S, Ghaffari M, Moztarzadeh F and Mozafari M: Corneal repair and regeneration: Current concepts and future directions. Front Bioeng Biotechnol 7: 135, 2019.

59. van Essen TH, van Zijl L, Possemiers T, Mulder AA, Zwart SJ, Chou CH, Lin CC, Lai HJ, Luyten GPM, Tassignon MJ, et al: Biocompatibility of a fish scale-derived artificial cornea: Cytotoxicity, cellular adhesion and phenotype, and in vivo immunogenicity. Biomaterials 81: 36-45, 2016.

60. Krishnan S, Sekar S, Katheem MF, Krishnakumar S and Sastry TP: Fish scale collagen-a novel material for corneal tissue engineering. Artif Organs 36: 829-835, 2012.

61. Bordoni M, Scarian E, Rey F, Gagliardi S, Carelli S, Pansarasa $\mathrm{O}$ and Cereda $\mathrm{C}$ : Biomaterials in neurodegenerative disorders: A promising therapeutic approach. Int J Mol Sci 21: $3243,2020$. 
62. Papadimitriou L, Manganas P, Ranella A and Stratakis E: Biofabrication for neural tissue engineering applications. Mater Today Bio 6: 100043, 2020.

63. Iwashita M, Ohta H, Fujisawa T, Cho M, Ikeya M, Kidoaki S and Kosodo Y: Brain-stiffness-mimicking tilapia collagen gel promotes the induction of dorsal cortical neurons from human pluripotent stem cells. Sci Rep 9: 3068, 2019.

64. Suzuki A, Kato H, Kawakami T, Kodama Y, Shiozawa M, Kuwae H, Miwa K, Hoshikawa E, Haga K, Shiomi A, et al Development of microstructured fish scale collagen scaffolds to manufacture a tissue-engineered oral mucosa equivalent. J Biomater Sci Polym Ed 31: 578-600, 2020.

65. Percival SL, McCarty S, Hunt JA and Woods EJ: The effects of $\mathrm{pH}$ on wound healing, biofilms, and antimicrobial efficacy. Wound Repair Regen 22: 174-186, 2014

66. Krzyszczyk P, Schloss R, Palmer A and Berthiaume F: The role of macrophages in acute and chronic wound healing and interventions to promote Pro-wound healing phenotypes. Front Physiol 9: 419, 2018

67. Hesketh M, Sahin KB, West ZE and Murray RZ: Macrophage phenotypes regulate scar formation and chronic wound healing. Int J Mol Sci 18: 1545, 2017.

68. Feng $X, X u$ W, Li Z, Song W, Ding J and Chen X: Immunomodulatory Nanosystems. Adv Sci (Weinh) 6: 1900101, 2019.

69. Liu C and Sun J: Osteogenically differentiated mesenchymal stem cells induced by hydrolyzed fish collagen maintain their immunomodulatory effects. Life Sci 238: 116970, 2019.

70. Pers YM, Bony C, Duroux-Richard I, Bernard L, Maumus M, Assou S, Barry F, Jorgensen C and Noël D: miR-155 Contributes to the immunoregulatory function of human mesenchymal stem cells. Front Immunol 12: 624024, 2021.

71. Abdi R, Fiorina $\mathrm{P}$, Adra $\mathrm{CN}$, Atkinson $\mathrm{M}$ and Sayegh $\mathrm{MH}$ : Immunomodulation by mesenchymal stem cells: A potential therapeutic strategy for type 1 diabetes. Diabetes 57: 1759-1767, 2008.

72. Chávez-Galán L, Olleros ML, Vesin D and Garcia I: Much more than M1 and M2 macrophages, there are also CD169(+) and TCR(+) macrophages. Front Immunol 6: 263, 2015.

73. Glenn JD and Whartenby KA: Mesenchymal stem cells: Emerging mechanisms of immunomodulation and therapy. World J Stem Cells 6: 526-539, 2014.

74. Liu Y, Yin Z, Zhang R, Yan K, Chen L, Chen F, Huang W, Lv B, Sun $C$ and Jiang X: MSCs inhibit bone marrow-derived DC maturation and function through the release of TSG-6. Biochem Biophys Res Commun 450: 1409-1415, 2014.

75. Spaggiari GM, Capobianco A, Abdelrazik H, Becchetti F, Mingari MC and Moretta L: Mesenchymal stem cells inhibit natural killer-cell proliferation, cytotoxicity, and cytokine production: Role of indoleamine 2,3-dioxygenase and prostaglandin E2. Blood 111: 1327-1333, 2008.

76. Aggarwal S and Pittenger MF: Human mesenchymal stem cells modulate allogeneic immune cell responses. Blood 105: 1815-22, 2005.

77. Glennie S, Soeiro I, Dyson PJ, Lam EW and Dazzi F: Bone marrow mesenchymal stem cells induce division arrest anergy of activated T cells. Blood 105: 2821-2827, 2005.

78. Asari S, Itakura S, Ferreri K, Liu CP, Kuroda Y, Kandeel F and Mullen Y: Mesenchymal stem cells suppress B-cell terminal differentiation. Exp Hematol 37: 604-615, 2009.

79. La Rocca G, Lo Iacono M, Corsello T, Corrao S, Farina F and Anzalone R: Human Wharton's jelly mesenchymal stem cells maintain the expression of key immunomodulatory molecules when subjected to osteogenic, adipogenic and chondrogenic differentiation in vitro: New perspectives for cellular therapy. Curr Stem Cell Res Ther 8: 100-113, 2013.

80. Liu C and Sun J: Impact of Marine-based biomaterials on the immunoregulatory properties of bone marrow-derived mesenchymal stem cells: Potential Use of Fish Collagen in Bone Tissue Engineering. ACS Omega 5: 28360-28368, 2020.
81. Ouyang L, Cao J, Dai Q and Qiu D: New insight of Immuno-engineering in osteoimmunomodulation for bone regeneration. Regen Ther 18: 24-29, 2021.

82. Liu C and Sun J: Modulation of the secretion of mesenchymal stem cell immunoregulatory factors by hydrolyzed fish collagen. Exp Ther Med 20: 375-384, 2020.

83. Hernández-Rangel A and Martin-Martinez ES: Collagen based electrospun materials for skin wounds treatment. J Biomed Mater Res A: Feb 27, 2021 (Epub ahead of print).

84. Shen XR, Chen XL, Xie HX, He Y, Chen W, Luo Q, Yuan WH, Tang X, Hou DY, Jiang DW and Wang QR: Beneficial effects of a novel shark-skin collagen dressing for the promotion of seawater immersion wound healing. Mil Med Res 4: 33, 2017.

85. Vigneswari S, Murugaiyah V, Kaur G, Abdul Khalil HP and Amirul AA: Biomacromolecule immobilization: Grafting of fish-scale collagen peptides onto aminolyzed P(3HB-co-4HB) scaffolds as a potential wound dressing. Biomed Mater 11: $055009,2016$.

86. Muthukumar T, Prabu P, Ghosh K and Sastry TP: Fish scale collagen sponge incorporated with Macrotyloma uniflorum plant extract as a possible wound/burn dressing material. Colloids Surf B Biointerfaces 113: 207-212, 2014.

87. Michalska-Sionkowska M, Warżýnska O Kaczmarek-Szczepańska B, Łukowicz K, Osyczka AM and Walczak M: Preparation and characterization of fish skin collagen material modified with $\beta$-glucan as potential wound dressing. Materials (Basel) 14: 1322, 2021.

88. Hartinger JM, Lukáč P, Mlček M, Popková M, Suchý T, Supová M, Chlup H, Horný L, Závora J, Adámková V, et al: Rifampin-releasing Triple-layer cross-linked fresh water fish collagen sponges as wound dressings. Biomed Res Int 2020: 3841861, 2020.

89. Feng X, Zhang X, Li S, Zheng Y, Shi X, Li F, Guo S and Yang J: Preparation of aminated fish scale collagen and oxidized sodium alginate hybrid hydrogel for enhanced full-thickness wound healing. Int J Biol Macromol 164: 626-637, 2020.

90. Ge B, Wang H, Li J, Liu H, Yin Y, Zhang $\mathrm{N}$ and Qin S: Comprehensive assessment of Nile Tilapia Skin (Oreochromis niloticus) collagen hydrogels for wound dressings. Mar Drugs 18: 178, 2020

91. Gómez-Guillén MC, Giménez B, López-Caballero ME and Montero MP: Functional and bioactive properties of collagen and gelatin from alternative sources: A review. Food Hydrocoll 25: 1813-1827, 2011.

92. Barczyk M, Carracedo S and Gullberg D: Integrins. Cell Tissue Res 339: 269-280, 2010

93. Sewing J, Klinger M and Notbohm H: Jellyfish collagen matrices conserve the chondrogenic phenotype in two- and three-dimensional collagen matrices. J Tissue Eng Regen Med 11: 916-925, 2017.

94. Chiu LH, Lai WF, Chang SF, Wong CC, Fan CY, Fang CL and Tsai YH: The effect of type II collagen on MSC osteogenic differentiation and bone defect repair. Biomaterials 35: 2680-2691, 2014.

95. Cho JK, Jin YG, Rha SJ, Kim SJ and Hwang JH: Biochemical characteristics of four marine fish skins in Korea. Food Chem 159: 200-207, 2014

96. Liu C and Sun J: The potential use of fish collagen as a new functional materials due to its good immune-compatibility. J Physics Conf Series 1605: 012174, 2020.

This work is licensed under a Creative Commons Attribution-NonCommercial-NoDerivatives 4.0 International (CC BY-NC-ND 4.0) License. 